\title{
Global hemianaesthesia: a parietal perceptual distortion suggesting non-organic illness
}

\author{
PHILIP YAR NELL, ELDAD MELAMED, \\ A N D R U T H I L VER B E G \\ From the Department of Neurology, Hadassah-Hebrew University Hospital, Israel, \\ and Department of Neurology, Denver General Hospital, University of Colorado Medical Center, \\ Denver, Colorado, USA
}

SUMMARY In two patients, lesions in the non-dominant parietal lobe were associated with global anaesthesia to all sensory modalities affecting the opposite half of the body. A striking inconsistency existed between the complaints of limb anaesthesia and the grossly preserved motor and postural control of the involved limb. The gross discrepancy between complaint and functional ability prompted early consideration of a "non-organic" diagnosis. It is suggested that this unusual sensory deficit may be due to distorted perception of somatosensory stimuli, representing another disorder of body schema associated with parietal lobe lesions. The diagnosis of non-organic illness may then be avoided by focusing on a search for parietal disease.

Parietal lobe lesions may present with dramatic perceptual disturbances such as anosognosia and hemiasomatognosia. The patient may deny an obvious hemiplegia or may even deny the existence of one half of his body. These disturbances are found most common in lesions of the nondominant parietal lobe (Critchley, 1966, Fredericks, 1969; Jewesburg, 1969). However, it is a clinical axiom that parietal lobe lesions do not cause a total hemisensory loss (DeJong, 1967; Jewesburg, 1969). Such a global sensory deficit may be observed in thalamic area lesions (DeJong, 1967; Brain and Walton, 1969; Adams and Victor, 1977) or in non-organic illness such as hysteria or hypersuggestible states (Spillane, 1975; Adams and Victor, 1977).

Two patients with non-dominant parietal lobe lesions who exhibited total hemianaesthesia to both leminiscal and spinothalmic mediated sensations were studied. A most unusual aspect in these patients was that their dense hemianaesthesia was not associated with the expected sensory ataxia nor with limitation of motor function in the involved limbs. The gross discrepancy between the sensory loss intact function prompted initial consideration of non-organic illness. We suggest that, in some

\footnotetext{
Address for reprint requests: Dr Philip R. Yarnell, Neurology Department, Denver General Hospital, W. 8th Avenue and Cherokee Street, Denver, Colorado 80204, USA.

Accepted 11 May 1978
}

patients, this discrepancy represents a parietal perceptual distortion with an altered hemicorporeal sensation being misperceived as a hemianaesthesia.

\section{Case reports}

\section{PATIENT 1}

A 53 year old, right handed, well-educated woman with a past history of rheumatic fever and transient cardiac arrythmias had the sudden onset of a momentary pressure sensation in her head while washing dishes. She then was unable to feel her left hand, and had the bizarre sensation that her left hand was not hers. She sat down on a chair, but fell on attempting to stand because she could not control her left side.

On admission the patient claimed a total left sided anaesthesia. Pinprick, touch, and temperature sensation were all absent on the left face, limbs, and hemitrunk. Two point discrimination and stereognosis were untestable in the presence of anaesthesia. Vibration was absent in the left extremities but felt equally on the sternum and forehead. Joint position sensation was absent in the left upper and lower extremities. She could not distinguish movement of any of her fingers, toes, wrist, or ankle even on extreme displacement of the examined joint. Despite this severe hemisensory loss there was no functional impairment in the left extremities, and she could perform fine 
finger movements perfectly. She could use her left hand to light, hold, and smoke a cigarette, to button her shirt, and to type without any trace of clumsiness. Rapid alternating movements and finger-to-nose test were normal without clumsiness or tremor. There was no dysmetria. Even without visual assistance she could find her fingers and hand in space and maintain a fixed hand and finger posture without any adventitious movements. Spontaneous gestures were performed normally with the hand. Despite the sensory complaints in her left leg, her gait was normal with no trace of ataxia. Heel-to-shin as well as the Romberg tests were normal. These findings were consistent on repeated examinations and persisted even after the discrepancy between sensory loss and intact motor function was pointed out to the patient. Cranial nerves, motor system, and deep tendon reflexes were all normal. Clinical tests of right hemispheric cognitive junctions such as sketching three dimensional objects, clock and map drawing, and dressing praxis were intact. She scored at the lower normal range on the Benton Facial Recognition test and on Koh's Blocks and Object Assembly tests.

There were no cardiac arrythmias, and the general physical examination was not contributory. The electroencephalogram and cerebrospinal fluid were normal. The diagnosis of hysteria was considered. However, a ${ }^{99} \mathrm{Tc}$ radionuclide brain image revealed a wedge-shaped area of increased uptake in the right parietal cortical area (Figure). The patient's complaints of total left hemianaesthesia improved only slightly during her time in hospital. At 10 days there was still complete subjective lack of joint position sense, and the patient was even uncertain whether the joints had moved at all. Interestingly, when asked to "guess" finger position, she "guessed" correctly consistently. The patient reported that objects often dropped from her left hand when she was not concentrating.

During the following weeks there was some improvement, but the patient still had a left hypaesthesia to spinothalamic and leminiscal mediated sensations. She noted difficulties in finding objects in her purse and in evaluating weights of objects. She claimed an inability to use her left hand meaningfully unless she attended specifically to it. Four months after the cerebral insult, her sensory complaints had subsided and she became symptom-free. Follow-up radionuclide images had become normal.

PATIENT 2

An 18 year old, right handed man felt paraesthesiae in his left hand immediately followed by tonic posturing while riding his bicycle. He fell and had a generalised seizure. Two days later a similar episode occurred. During those days, the patient noted that objects were falling out of his left hand and he could not keep his sandal on his left foot. On admission, the patient was well oriented, with-

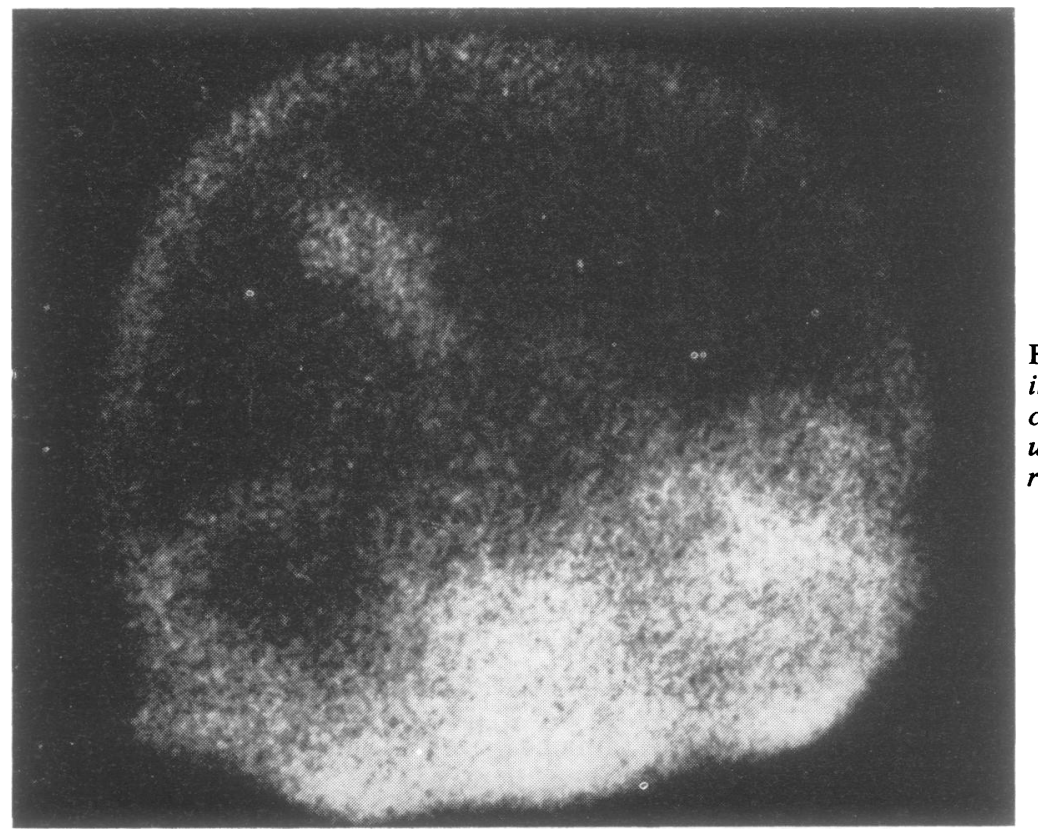

Figure Patient 1: radionuclide image, right lateral, showing the cortical parietal wedge-shaped uptake. This subsequently resolved in three months. 
out language or memory impairment. He had a dense anaesthesia to pinprick, touch, and temperature on the left side of the face, trunk, and limbs. Two point discrimination and stereognosis could not be assessed because of the anaesthesia. He denied knowing the position of any of his left fingers, toes, wrist, and ankle, even with extreme movements of the joint. Sensation of vibration was absent in the left extremities but felt equally on the sternum and forehead. Motor and reflex examinations were normal. There was a gross discrepancy between the subjective dense hemisensory loss and intact functional abilities. Despite the marked hemisensory defect, he could touch his nose and localise his left hand and fingers in space without visual assistance. There were no adventitious movements, and he used his left hand in a perfectly normal manner. Rapid alternating movements were normal. Furthermore, despite the loss of joint position sensation in his leg, his gait was undisturbed. Heel-to-shin movements were also unimpaired. These findings were consistent on repeated examinations and persisted even after the discrepancy was demonstrated to the patient. Since he had a focal seizure indicative of an organic brain lesion, "psychological elaboration or overlay" was the mechanism originally considered to explain the patient's exaggerated sensory complaints.

A carotid angiogram demonstrated a large right parietal avascular mass. Surgery was performed shortly thereafter, and a mixed glioma in the parietal region was partially resected. The patient underwent a course of radiation therapy, but had a progressive decline, and died nine months later. Permission for necropsy was refused.

\section{Discussion}

These two patients with non-dominant parietal lobe lesions had global anaesthesia to all sensory modalities, affecting the contralateral half of the body. Such sensory abnormalities are most unusual in lesions of the parietal lobe (DeJong, 1967). Most often parietal lesions are associated with a variable contralateral sensory loss. There is usually a diminution of spinothalamic and leminiscal modalities rather than a total loss (DeJong, 1967). The sensory deficit is often so mild that patients may be unaware of its existence (Critchley, 1966). Moreover, the sensory loss may not be uniformly hemicorporeal (Fredericks, 1969). The hand may be often disproportionately involved with a characteristic sensory loss of distal distribution as in root or peripheral nerve lesions. At other times the sensory loss may be limited to small scattered zones such as the circumoral area or the radial side of the hand (Critchley, 1966; Adams and Victor, 1977). Although, when present, these partial hemianaesthesias are the rule in parietal lobe disease. Critchley (1966) cites a case where the sensory loss was global and affected all modalities. He also cites another patient with brain trauma in whom a global hemianaesthesia evolved into a more usual parietal sensory syndrome.

Dense hemianaesthesia affecting all sensory modalities may be caused by thalamic and capsular lesions (DeJong, 1967; Brain and Walton, 1969; Adams and Victor, 1977). However, patients with such an anaesthesia including joint position sense loss are unable to perform skilled or fine movements, since intact motor function is dependent on normal somatosensory input (DeJong, 1967; Spillane, 1975). By contrast, in our patients, the global hemianaesthesia did not limit their ability to maintain finger dexterity, motor manipulative skills, spatial postural control, and normal gait. Therefore, it is unlikely that these patients had an extension of the parietal lesion into deeper subcortical sensory structures.

When such marked discrepancies exist between the dramatic sensory complaints and the intact functional abilities, non-organic illness such as hysteria must be considered. Because of this discrepancy, our patients' complaints were initially judged non-organic due either to hysteria in patient 1 , or to psychological elaboration in patient 2. Critchley (1966), has noted that in parietal lobe disease the differential diagnosis from hysteria was most difficult in "those cases where there is a hysterical elaboration of organic parietal manifestations."

Alternatively, the global hemianaesthesia in these two patients may represent a perceptual distortion caused by their parietal lesions. Parietal lobe disease is known to be associated with perceptual aberrations such as extinction, neglect, and disorders of body schema as summarised by Fredericks (1969) and Jewesburg (1969). Sensory extinction is defined as the inability to perceive sensation on one side of the body when identical areas on the two sides are stimulated simultaneously. Such a mechanism does not apply in these patients since they did not even perceive sensation on unilateral stimulation. In patients with parietal neglect, a spectrum of behavioural symptoms may be observed ranging from inattention to complete denial of obvious objective illness. However, neglect cannot account for the sensory syndrome in these patients since they did not deny their symptoms but rather complained of the global hemianaesthesia. In spite of their complaints 
of dense hemianaesthesia, it is possible that these patients had only a minor sensory deficit or no objective primary sensory impairment at all. Their sensory impairment, particularly in the absence of the expected abnormalities in co-ordinative motor functions, may represent another type of disorder of the body schema. One may consider our patients' sensory symptomatology as an obverse of anosognosia. Instead of denial of an obvious hemicorporeal defect, there is an exaggeration or misinterpretation of a lesser deficit. In this way, the marked discrepancy between the sensory complaints and intact functional abilities may be understood as a parietal misperception. The diagnosis of non-organic illness may then be avoided in such patients by focusing on a search for parietal lobe disease.

We thank Dr M. Globus for referring patient 1, and Shlomo Bentin, MSc, for performing formal psychological testing on this patient.

\section{References}

Adams, R. D., and Victor, M. (1977). Principles of Neurology, Chapter 9. McGraw-Hill: New York.

Brain, W. R., and Walton, J. M. (1969). Brain's Diseases of the Nervous System, 7th edition, chapter 1. Oxford University Press: London.

Critchley, M. (1966). The Parietal Lobes, Facsimile of 1953 edition, chapters 4, 8, 10. Hafner Publishing Company: New York.

DeJong, R. N. (1967). The Neurologic Examination, 3rd edition, chapter 8. Harper and Row: New York.

Fredericks, J. A. M. (1969). Disorders of body schema. In Handbook of Clinical Neurology, vol. 4, chapter 11. Edited by P. J. Vinken and G. W. Bruyn. John Wiley and Sons: New York.

Jewesburg, E. C. O. (1969). Parietal lobe syndrome. In Handbook of Clinical Neurology, vol. 2, chapter 21. Edited by P. J. Vinken and G. W. Bruyn. John Wiley and Sons: New York.

Spillane, J. D. (1975). An Atlas of Clinical Neurology, 2nd edition, chapter 13. Oxford University Press: London. 